\title{
Methylxanthines induce structural and functional alterations of the cardiac system in zebrafish embryos
}

\author{
Ram Manohar Basnet ${ }^{1 *}$, Daniela Zizioli ${ }^{1}$, Michela Guarienti ${ }^{1}$, Dario Finazzi ${ }^{1,2}$ and Maurizio Memo ${ }^{1}$
}

\begin{abstract}
Background: Zebrafish embryos are emerging as a model for pharmacological and toxicological studies. We used zebrafish embryos to study the general toxicity and cardiovascular effects of eight methylxanthines: aminophylline, caffeine, diprophylline, doxofylline, etophylline, 3-isobutyl-1-methylxanthine (IBMX), pentoxifylline and theophylline.

Methods: Microinjections of the eight methylxanthines were performed in 1-2 cell stage zebrafish embryos and the general toxicity and cardiovascular effects were analyzed at different time points. Embryotoxicity and teratogenicity were evaluated to understand the general toxicity of these compounds. Structural and functional alterations of the heart were evaluated to assess the cardiovascular effects.

Results: Our results showed different activity patterns of the methylxanthines drugs. Caffeine, IBMX, pentoxifylline and theophylline were highly embryotoxic and teratogenic; aminophylline, doxofylline and etophylline were embryotoxic and teratogenic only at higher doses, and diprophylline showed a minimal (<10\%) embryotoxicity and teratogenicity. Most of these drugs induced structural alteration of the heart in $20-40 \%$ of the injected embryos with the maximum dose. This structural alteration was fatal with the embryos ultimately dying within $120 \mathrm{hpf}$. All the drugs induced a transient increase in heart rate at $48 \mathrm{hpf}$ which returned to baseline within 96 hpf. This functional effect of methylxanthines showed similarity to the studies done in humans and other vertebrates.

Conclusion: Our results indicate the potential toxicity and teratogenicity of different methylxanthines in the embryos during embryonic development, the most sensitive period of life. Although interspecies differences need to be considered before drawing any conclusion, our study elucidated that a single exposure of methylxanthines at therapeutic range could induce cardiac dysfunction besides causing embryotoxicity and teratogenicity. Of all the drugs, diprophylline appeared to be safer, with lower degree of embryotoxicity, teratogenicity and cardiac toxicity as compared to other methylxanthines.
\end{abstract}

Keywords: Embryotoxicity, Teratogenicity, Methylxanthines, Heart rate, Cardiac dysfunction

\section{Background}

Methylxanthines are one of the widely consumed substances in the world $[1,2]$. The sources of methylxanthines include tea, coffee, chocolate, energy drinks and drugs [1]. They can be naturally occurring, such as caffeine, theobromine and theophylline, or synthetic derivatives, such as diprophylline and pentoxifylline $[3,4]$. Beverages rich in

\footnotetext{
*Correspondence: rmb.basnet@gmail.com; r.basnet@unibs.it

${ }^{1}$ Department of Molecular and Translational Medicine, University of Brescia,

Viale Europa, 11, 25123 Brescia, Italy

Full list of author information is available at the end of the article
}

methylxanthines have been consumed in different cultures across centuries $[1,5,6]$. These substances are also used for the treatment of diverse medical conditions such as asthma, chronic obstructive pulmonary disease (COPD), peripheral vascular disease, obesity, hyperlipidemia and apnea of prematurity [3, 7-13]. Therefore, these compounds are regularly ingested in a daily basis through various beverages and drugs. All these drugs have similar mechanism of action; they act via inhibition of phosphodiesterase enzyme, antagonism of adenosine and $\mathrm{GABA}_{\mathrm{A}}$ receptors, and blocking of the calcium channels $[1,3,5]$. 
The human exposure to methylxanthines begins as early as gestation and continues throughout the life [14]. Although in human, fatal cases of methylxanthine toxicity with theophylline, aminophylline [15], caffeine [16, 17] and pentoxifylline [18] from accidental overdose and suicide have been reported, they are rare and mostly iatrogenic [14]. Rather, the therapeutic use of methylxanthines has been limited by their life threatening adverse effects, such as cardiac arrhythmias and intractable seizures [19]. For instance, theophylline has been replaced to fourth line of therapy in the treatment of chronic obstructive pulmonary disease as it causes arrhythmia and seizures. Also other methylxanthines, like aminophylline and caffeine, have been implicated in several types of cardiac arrhythmia. As cardiotoxicity is one of the principal reasons for the drug withdrawal [20], prior information of the cardiotoxic property of these drugs could save time and money in the drug development process.

Cardiac toxicity and hemodynamic perturbations are two major aspects of methylxanthine toxicity [21]. Tachydysrhythmias which includes sinus tachycardia and premature ventricular contraction are the most common dysrhythmia observed with methylxanthines toxicity [22]. They also cause widened pulse pressure, hypotension, myocardial ischemia and infarction [23]. The complexity and relevance of the observed cardiac toxicity strengthen the importance of further studies of methylxanthines induced cardiotoxicity.

The purpose of our study was twofold. On one hand, we wanted to study the general and cardiac toxicity of methylxanthines. At the same time, we also wanted to study the teratogenicity of these drugs. To achieve our goals, we employed zebrafish embryos which might provide us the insight regarding the toxicity and teratogenicity of these drugs simultaneously.

Zebrafish are emerging as a predictive animal model for assessing the in vivo effects and toxicity of various compounds and drugs [24-27] with numerous studies showing overwhelming similarity between mammalian and zebrafish toxicity [20,24, 27, 28]. Moreover, recent evidences have also shown a high degree of similarity between the pharmacological responses of zebrafish and human to known cardiotoxins [20]. In addition to the general advantages over other animal models such as transparent embryos, external fertilization, high fecundity, economical and easy manipulation [29], zebrafish embryos have features which favor its use in the study of drug induced cardiovascular effects. They have a simple cardiac and vascular system, and the molecular mechanisms underlying vascular tree development strongly resembles those of higher vertebrates, showing a high degree of anatomical and functional conservation [30]. At $48 \mathrm{hpf}$, the zebrafish cardiovascular system is fully functional with a complex repertoire of ion channels and metabolic processes already developed [31]. Like mammals, they possess a pacemaker which controls heart beat, the blood is collected by the atrium and is pumped throughout the body by the ventricle. The transparency of zebrafish embryos makes it easy to study the heart rate and rhythm, cardiac morphology and blood circulation [31, 32]. Furthermore, the availability of transgenic embryos has made it easy to study the in vivo effects of cardiovascular system effectively.

In this study, we investigated the general and cardiac toxicity of eight methylxanthines compounds: aminophylline, caffeine, diprophylline, doxofylline, etophylline, 3-isobutyl-1methylxanthine (IBMX), pentoxifylline and theophylline in zebrafish embryos. These methylxanthines were selected based on their presence in commonly consumed beverages such as coffee, tea and mate, therapeutic uses in diverse medical conditions such as COPD, peripheral vascular disorders, hyperlipidemia and apnoea of prematurity and their water solubility. Zebrafish embryos were microinjected with each methylxanthine compound with five predetermined graded doses of each drug into the yolk of 1-2 cell zebrafish embryos. We firstly studied the general toxicity caused by these drugs in zebrafish embryos. Cumulative mortality induced by these drugs was calculated after 72 hpf. The phenotype of surviving/alive embryos was classified as normal, mild and severe based on the observance of morphological defects. Secondly, we studied the cardiac toxicity of methylxanthines taking into account both the structural and functional aspects of the heart. Our study aims to provide the insight into the cardiac teratogenicity within the realm of general and cardiac toxicity of methylxanthines.

\section{Methods}

Drugs

Eight methylxanthine drugs were used in the study: aminophylline, caffeine, diprophylline, etophylline, IBMX, theophylline from Sigma and doxofylline and pentoxifylline from Abcam. Metoprolol, a $\beta$-blocker used for the cardiovascular study was obtained from Sigma.

\section{Zebrafish maintenance and collection of eggs}

Zebrafish were maintained and used in accordance with the Italian and European rules on animal use following protocols approved by the local Committee (OPBA protocol nr 211B5.24) and authorized by the Ministry of Health (authorization number 393/2017-PR).

Adult $\mathrm{AB}$ wild type and transgenic line tg (Bmp:EGFP) [33] zebrafish were raised and maintained in 14: $10 \mathrm{~h}$ light: dark cycle at $28^{\circ} \mathrm{C}$ in a circulating system maintained at $\mathrm{pH} 7.0$ and conductivity between 400 and $500 \mu$ s. Fishes were fed three time per day with a combination of granular food (from special diet services, SDS, Witham, UK) in the morning and evening and 
artemia freshly prepared in the laboratory in the afternoon (cysts bought from SDS, Witham, UK).

Adult zebrafish were used for egg production. Male and female were put in the breeding tank overnight and next morning fresh spawned embryos were collected. Any unfertilized eggs, dead or bad embryos were removed and the good quality embryos at 1-2 cell stage were selected for microinjection.

\section{Microinjection}

Zebrafish embryos were injected with five different concentrations of eight methylxanthines at 1-2 cell stage. The total volume of injection was $5 \mathrm{~nL} / \mathrm{embryo}$ and a minimum of 100 embryos were injected for each concentration. Each compound was co-injected with $0.05 \%$ phenol red as a tracer. The total quantity of drug injected per embryo was obtained by calculating the total amount of drug in nanogram $(\mathrm{ng})$ present in $5 \mathrm{~nL}$ of all the five injected concentrations of each drug. The doses of the drugs injected per embryo ranged from $0.25 \mathrm{ng}$ to $20 \mathrm{ng}$ (Table 1). 3, 4-dichloroaniline (DCA) was used as positive control and embryos injected with $0.05 \%$ phenol red in sterile water acted as negative control. The non-injected embryos were used as a control to confirm that the needle pricking per se did not have any effects in the embryos. After microinjection, embryos were collected in Petri dish and maintained in fish water at $28{ }^{\circ} \mathrm{C}$ until further evaluation. Any defective or damaged embryos were discarded. The procedure was performed three times independently and the results are the mean \pm S.D. of three experiments.

\section{Evaluation of mortality and general morphology}

Mortality of the injected embryos was recorded at each time points of 24,48 and $72 \mathrm{hpf}$. The survival rates of each

Table 1 Doses of methylxanthines injected per embryo in nanogram

\begin{tabular}{llllll}
\hline Methylxanthines & \multicolumn{5}{l}{ Injected dose (ng/ embryo) } \\
\hline Aminophylline & 0.5 & 1.25 & $2.5^{*}$ & 3.75 & 5 \\
Caffeine & 0.25 & 0.5 & $0.75^{*}$ & 1.5 & 2.5 \\
Diprophylline & 1 & 2.5 & $5^{*}$ & 10 & 20 \\
Doxofylline & 1 & 2 & $3^{*}$ & 5 & 7.5 \\
Etophylline & 0.5 & 1 & $2^{*}$ & 3 & 5 \\
IBMX & 0.15 & 0.25 & $0.5^{*}$ & 0.75 & 1 \\
Pentoxifylline & 0.15 & 0.5 & $1^{*}$ & 2 & 3 \\
Theophylline & 0.25 & 0.5 & $1^{*}$ & 1.5 & 2.5 \\
Dichloroaniline & 0.015 & 0.05 & $0.1^{*}$ & & \\
Metoprolol & 0.005 & 0.01 & $0.018^{*}$ & 0.037 & 0.075 \\
\hline
\end{tabular}

The doses labeled with asterisk $\left(^{*}\right)$ were selected for the experiments in the transgenic tg (Bmp:EGFP) embryos to study the long term effect of methylxanthines in heart rate. Dichloroaniline was used as positive control in evaluation of mortality and general morphology, and metoprolol was used as internal control in evaluation of heart rate drugs was calculated and a dose-response survival graph was plotted at $72 \mathrm{hpf}$. A thorough evaluation of morphological endpoints from head to tail was performed at 48 and $72 \mathrm{hpf}$ (Table 2). The phenoype of the embryos was then classified into normal, mild or severe based on the abnormality of evaluated endpoints. The morphological evaluation and phenotype grading was performed under direct visualization with Leica MZ 16F Stereomicroscope (Germany).

\section{Evaluation of cardiovascular effects}

After performing microinjection as described above, structural cardiac defect and functional cardiac effects of the methylxanthine drugs were evaluated together with other cardiovascular endpoints.

\section{Structural cardiac defect}

At $48 \mathrm{hpf}$, embryos were evaluated for any structural cardiac abnormality and categorized into two groups: normal phenotype, i.e. embryos with no visible cardiac abnormality, and embryos with cardiac phenotype, i.e. embryos with visible structural cardiac abnormality. Then, the percentage of embryos with cardiac phenotype was calculated for each of the drugs. Furthermore, the cardiac phenotype embryos were graded into ' 3 ', '2' and ' 1 ', based on the criteria modified from Panzica-Kelly et al., 2010 [34].

Normal heart morphology with slightly smaller ventricle Atrium and ventricle were either compressed or severely enlarged and misshaped with no clear boundary between them

Atrium and ventricle were severely deficient, misshaped and not well 1 defined

The structural cardiac abnormality was identified under direct visualization of the heart of zebrafish embryos using Leica MZ 16F Stereomicroscope (Germany) at 80X magnification.

\section{Evaluation of cardiovascular endpoints}

In addition to the structural cardiac defects, we also evaluated the presence of several cardiovascular endpoints which included pericardial edema, decreased or absent blood flow, arrhythmia, hemorrhage and thrombosis. The total number of embryos developing each of these endpoints with all the eight methylxanthines was documented and the resulting percentage calculated for each of the endpoints at $48 \mathrm{hpf}$.

To see the fate of the embryos with cardiac defects beyond $72 \mathrm{hpf}$, one selected concentration of each of the eight compounds was injected into the embryos at 1 cell stage. The embryos with cardiac defects were then evaluated for mortality till $5 \mathrm{dpf}$. 


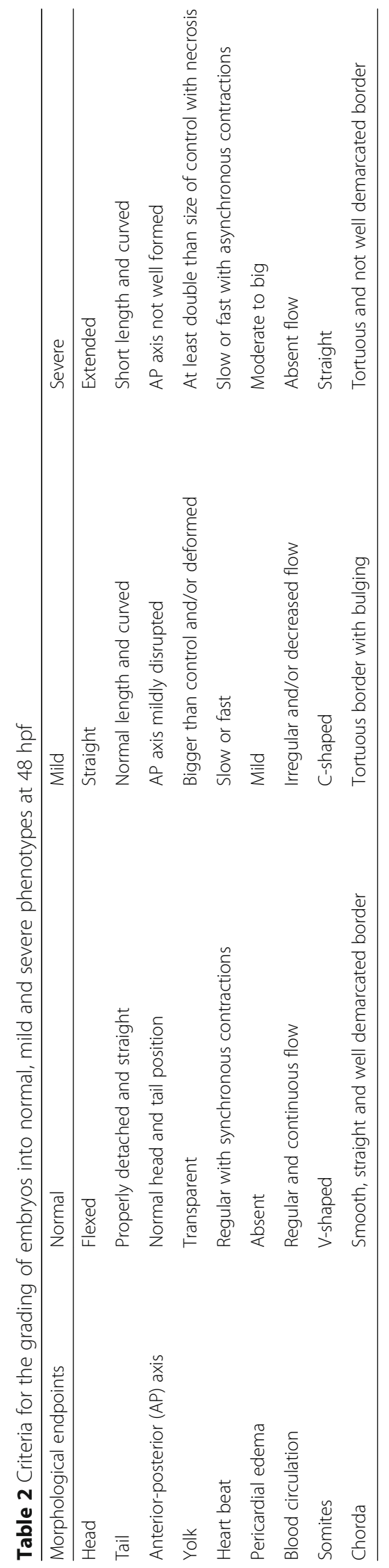




\section{Functional cardiac effect}

To study the functional cardiac effects of methylxanthines, the heart rate of normal looking embryos was measured at 48 hpf. Normal looking embryos implied the embryos with intact structural integrity including the normal cardiac strucuture and cardiovascular endpoints. For each drug, ten embryos were randomly selected from a pool of normal looking embryos and the heart beat of each of the embryos was counted manually for $30 \mathrm{~s}$ under Leica MZ16F stereomicroscope (Germany) in quiet condition and at optimal temperature. The heart beat per $30 \mathrm{~s}$ was multiplied by 2 to obtain the heart beat per minute i.e. heart rate. Metoprolol, a beta blocker which decreases the heart rate in human, was used as an internal control and embryos injected with sterile water was used as negative control.

To further understand if the functional cardiac effect was reversible or irreversible, we performed the same experiment in the $\operatorname{Tg}(B m p: E G F P)$ transgenic line with the embryos injected with one concentration of each drug. BMP transgenic line allows for the easy visualization of heartbeat, especially at 72 and $120 \mathrm{hpf}$ when the pigmentation might make it difficult to count the heart beat in the wild type embryos. The heart rate was measured starting from $48 \mathrm{hpf}$ till $120 \mathrm{hpf}$, at 24-h interval. The procedure for the measurement of heart beat was same as described earlier. However, for the measurement of heart beat at 72, 96 and $120 \mathrm{hpf}$, embryos were stabilized by briefly placing them for $10-15 \mathrm{~s}$ in low dose tricaine $(0.16 \mathrm{mg} / \mathrm{mL})$ before measuring the heart beat.

\section{Statistical analysis}

All the experiments were repeated at least three times and the data are reported in Mean \pm S.D. One way ANOVA with Dunnett's test was used for the comparison of the heart rate of the treated embryos with control. Statistical analysis was done in graph pad prism 5 (La Jolla, CA USA) with $p$ value $<0.05$ considered statistically significant.

\section{Results}

\section{Embryotoxicity}

As a first step in the characterization of the toxicity of methylxanthine compounds, we studied the lethality and phenotypic effects.

Five increasing doses/graded doses of eight methylxanthines: aminophylline, caffeine, diprophylline, doxofylline, etophylline, 3-isobutyl-1-methylxanthine (IBMX), pentoxifylline and theophylline; were injected into the yolk of 1-2 cell zebrafish embryos (Table 1) and the survival and phenotype analyzed at specific timepoints. The doses were selected in such a way that they fall within per oral therapeutic range in human. As the weight of zebrafish embryos is roughly $1.2 \mathrm{mg}$, administering $1 \mathrm{ng}$ of a drug in one embryo (1 $\mathrm{ng} /$ $1.2 \mathrm{mg}$ ) is approximately equal to $1 \mathrm{mg} / \mathrm{kg}$ of drug. Therefore, the doses of drugs used in microinjection were in the range of $1-20 \mathrm{mg} / \mathrm{kg}$ and most of these drugs have per oral therapeutic dose that falls within this range in humans.

Aminophylline, caffeine, doxofylline, IBMX, pentoxifylline and theophylline treated embryos showed dose dependent survival at $72 \mathrm{hpf}$, whereas the survival of diprophylline and etophylline treated embryos was independent of the doses (Fig. 1). IBMX was the most toxic/active compound: a large percentage of the embryos (58\%) showed mortality when injected with $1 \mathrm{ng}$ of the drug. Diprophylline was the least active methylxanthine; with more than $90 \%$ of embryos surviving at dose as high as $20 \mathrm{ng} / \mathrm{embryo}$. Pentoxifylline and caffeine also produced $>50 \%$ mortality, although they required higher doses than IBMX, i.e. $3 \mathrm{ng}$ and $2.5 \mathrm{ng}$ per embryo respectively to produce the equivalent mortality.

To simplify the comparison, we observed the dose at which there was a minimum of $20 \%$ mortality for each drug. Methylxanthines like IBMX, caffeine, pentoxifylline and theophylline were highly active with $<1 \mathrm{ng}$ needed to induce $20 \%$ mortaility. Aminophylline, etophylline and doxofylline were moderately active with 2.5-5 ng required to induce $20 \%$ mortality and diprophylline was a non toxic compound which didn't induce $20 \%$ mortality even with the highest dose of $20 \mathrm{ng}$. To further compare the toxicity of each individual compound, we calculated the lethal dose at which there was $50 \%$ mortality $\left(\mathrm{LD}_{50}\right)$ for each drug using linear interpolation and extrapolation. Our results showed that IBMX was the most toxic drug (LD50 $=0.91 \mathrm{ng})$ followed by caffeine $(2.4 \mathrm{ng})$, pentoxifylline (2.76 ng), theophylline (3.4 ng) and aminophylline (6.15 ng), etophylline (9.8 ng),doxofylline (29.3 ng) and diprophylline (167.5 ng).

General Teratogenicity and general morphological defects After evaluating the mortality, the alive embryos were observed for morphological defects at 48 and $72 \mathrm{hpf}$. The embryos with morphological defects were identified and classified into mild and severe phenotype based on the presence of different morphological endpoints (Table 2). In particular, embryos with defective heart formation, irregular heartbeat, decreased blood circulation, mild pericardial and yolk sac edema were categorized as mild phenotype. Embryos with perturbed anterior-posterior axis development with reduced tail detachment and poor formation of somites, defective heart formation, irregular heartbeat, absent blood circulation, severe pericardial and yolk sac edema were categorized as severe phenotype.

The percentage of embryos that showed morphological defects with the highest concentration of drug varied with each compound. While the lowest dose used produced morphological defects in $<10 \%$ of the embryos, the 


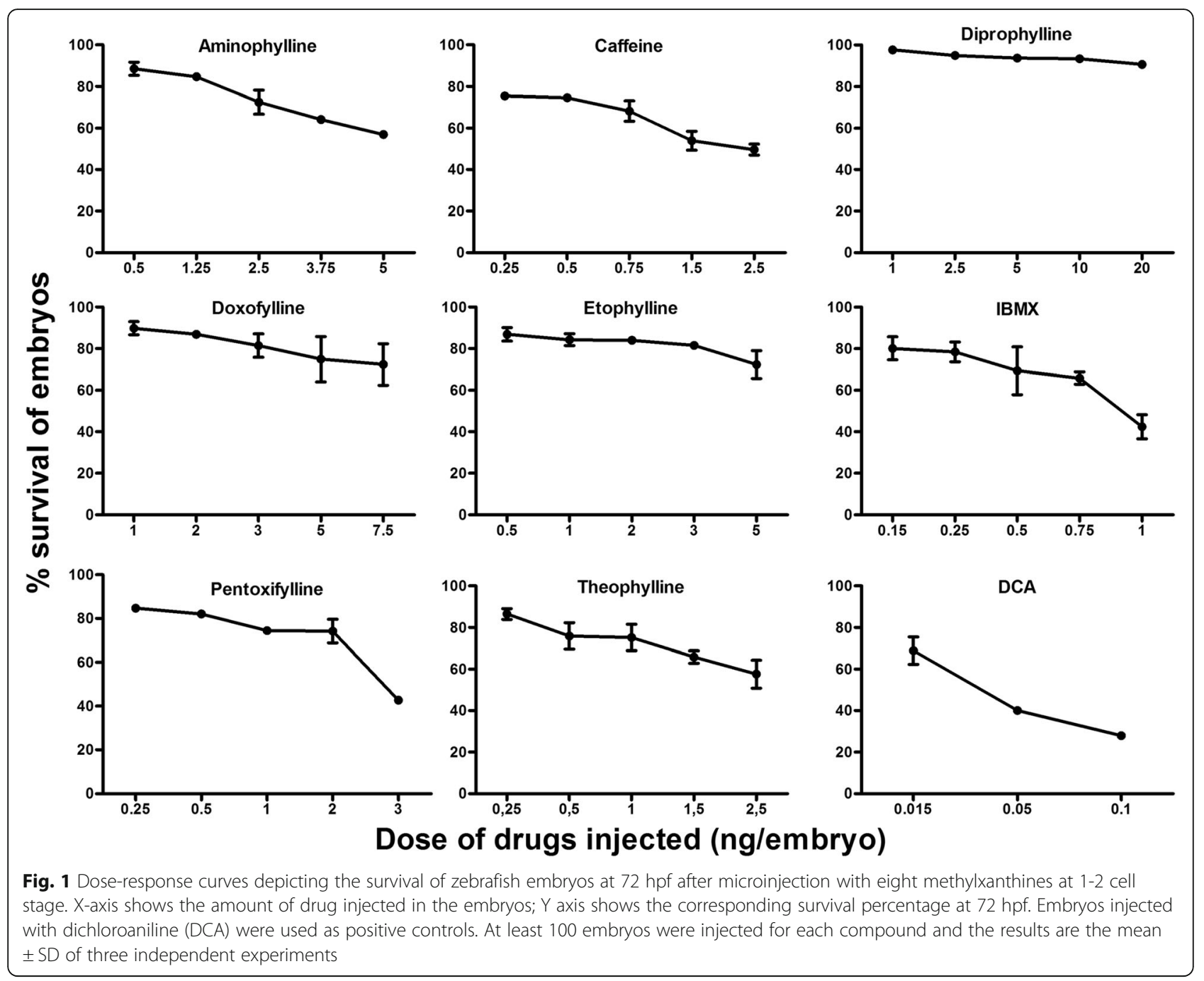

highest concentration produced morphological defects in $50 \%$ of embryos with $2.5 \mathrm{ng}$ of caffeine, $44 \%$ with $1 \mathrm{ng}$ of IBMX, 38\% with $3 \mathrm{ng}$ of pentoxifylline, 29\% with $5 \mathrm{ng}$ of aminophylline, $24 \%$ with $1 \mathrm{ng}$ of theophylline, 23\% with $7.5 \mathrm{ng}$ of doxofylline, $15 \%$ with $5 \mathrm{ng}$ of etophylline and $5 \%$ with $20 \mathrm{ng}$ of diprophylline. The embryos injected with the positive control DCA showed $47 \%$ morphological defects, while less than $1 \%$ of negative controls injected with sterile water showed morphological defects (Fig. 2). These embryos predominantly had pericardial edema, abnormal blood circulation, yolk sac edema and abnormal AP axis formation (Fig. 3).

For the comparison of the morphological defects, we looked into the dose required to induce morphological defects in at least $20 \%$ of surviving embryos for each drug. The results showed that caffeine, IBMX, pentoxifyline and theophylline required $\leq 1 \mathrm{ng}$ of drug; aminophylline and doxofylline required 3-5 ng of drug to induce the morphological defects in at least $20 \%$ of the embryos. However, diprophylline and etophylline didn't cause morphological defects in $20 \%$ of the embryos even with the highest doses of $20 \mathrm{ng}$ and $5 \mathrm{ng}$ respectively.

\section{Cardiac toxicity and Teratogenicity}

The cardiovascular effects of methylxanthines in zebrafish embryos were more extensively evaluated. Zebrafish embryos were injected with the same drugs and doses (see Table 1) at 1-2 cell stage. Embryos were then analyzed for the structural and functional alterations.

\section{Structural alteration}

Assessment of the structural alteration of the heart was performed at $48 \mathrm{hpf}$. The embryos showing structural cardiac defect were termed cardiac phenotype and embryos with normal cardiac morphology were termed normal phenotype. The total percentage of embryos with cardiac phenotype was calculated for each of the compounds (Fig. 4a). Each of the embryos with cardiac phenotype was 


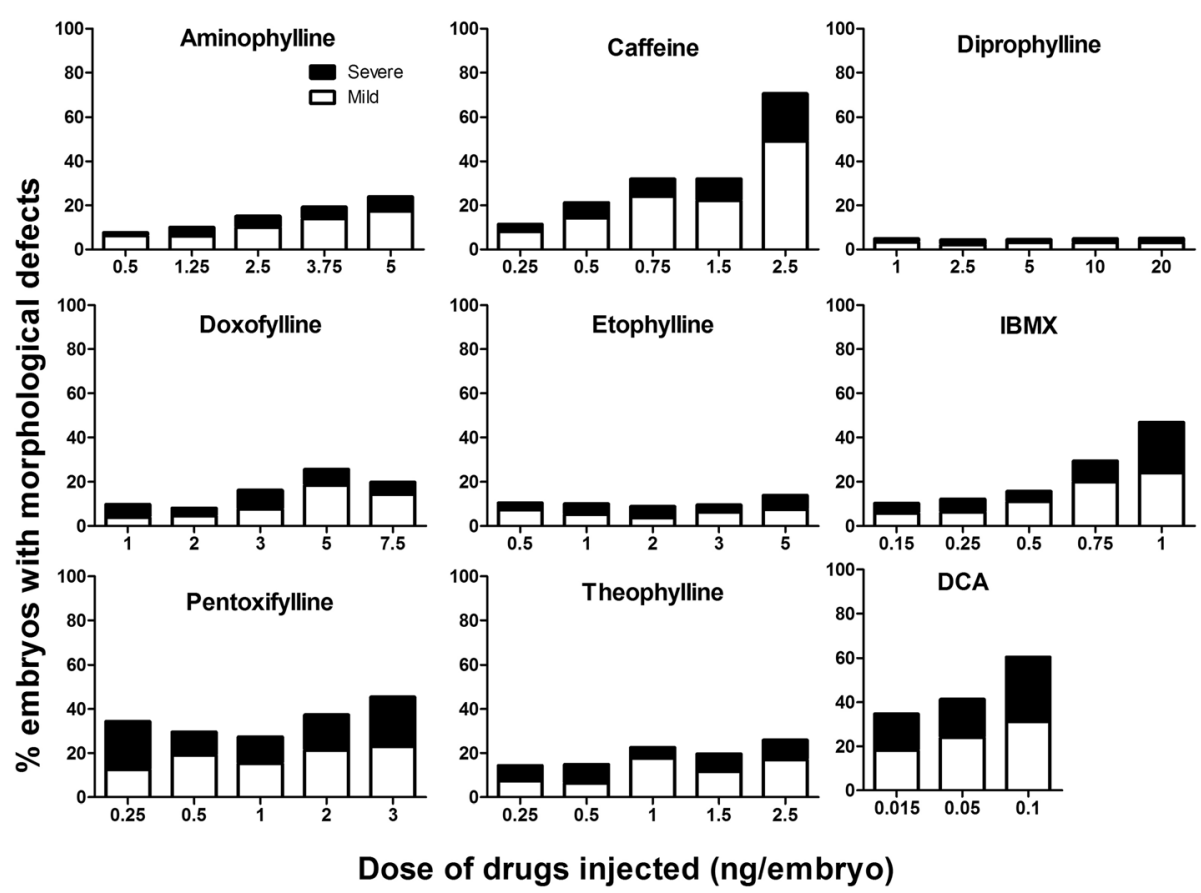

Fig. 2 Percentage of embryos showing morphological defects at $72 \mathrm{hpf}$ after microinjection with eight methylxanthines at 1-2 cell stage. For each drug, the percentage of embryos with mild (white bar) or severe (black bar) phenotype was assessed at each of the five tested concentrations. Embryos injected with dichloroaniline (DCA) were used as positive controls. At least 100 embryos were injected for each compound and the results are the mean \pm SD of three independent experiments

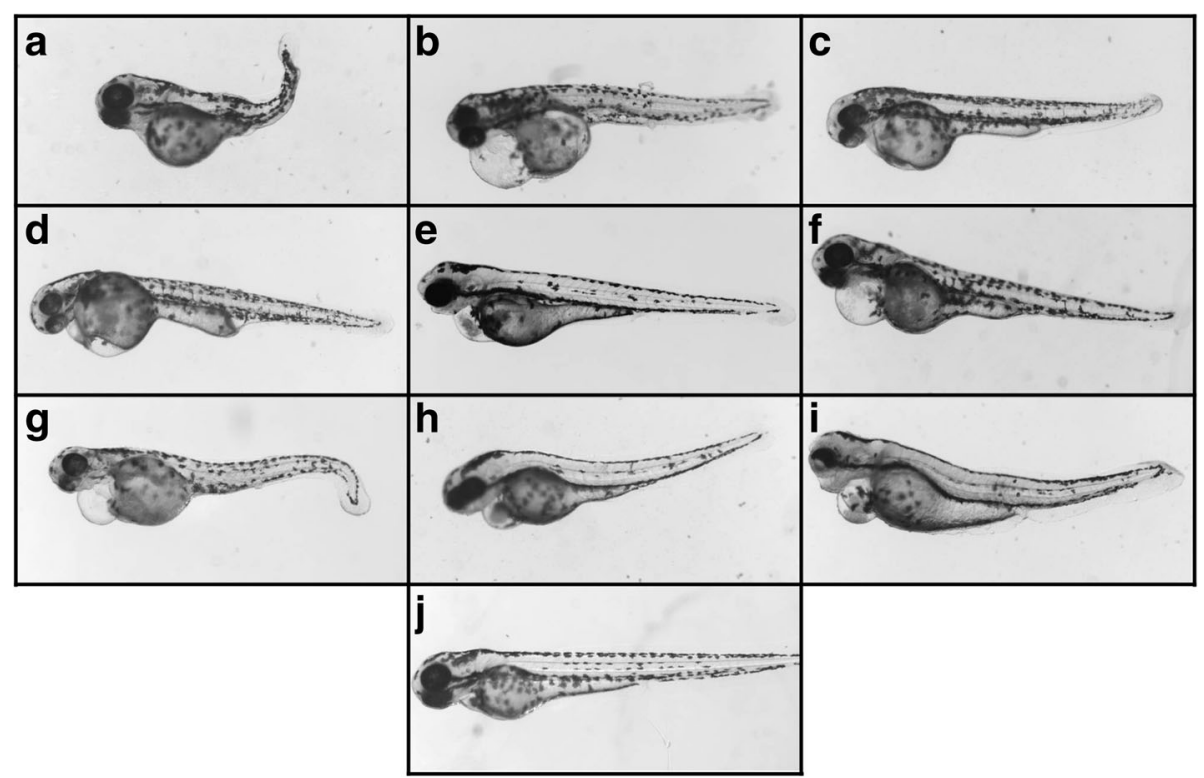

Fig. 3 Representative pictures of zebrafish embryos at 72 hpf injected with: a Aminophylline; b Caffeine; c Diprophylline; d Doxofylline; e Etophylline; $\mathbf{f} \mid \mathrm{BM} X ; \mathbf{g}$ Pentoxifylline; $\mathbf{h}$ Theophylline; $\mathbf{i}$ Dichloroaniline (positive control); and $\mathbf{j}$ Sterile water (negative control). The zebrafish embryos representative pictures were obtained with Leica MZ16F stereomicroscope(Germany) equipped with DFC 480 digital camera and LAS Leica Imaging software (Leica).Magnification 40X 
further assessed and graded based on the the criteria modified by the study of Panzica Kelly et al. [34]. The structural cardiac deformity in the embryos was scored as follows: 3 in case of normal heart morphology with slightly smaller ventricle, 2 if the atrium and ventricle were either compressed or severely enlarged and misshaped with no clear boundary between the atrium and ventricle and 1 if the atrium and ventricles were severely deficient, misshaped and not well defined.

Our results showed that with the exception of diprophylline and etophylline, all the other drugs induced cardiac phenotype in $20-40 \%$ of embryos with the highest dose. The minimum dose that caused at least $10 \%$ cardiac defects was $0.15 \mathrm{ng}$ for IBMX, $0.25 \mathrm{ng}$ for caffeine, pentoxifylline and theophylline, $1 \mathrm{ng}$ for doxofylline, $2.5 \mathrm{ng}$ for aminophylline and $5 \mathrm{ng}$ for etophylline. With diprophylline, the cardiac defect was present in less than $10 \%$ of the embryos even with the highest concentration of $20 \mathrm{ng}$. Further analysis of the embryos with cardiac phenotype showed that most of them had grade 2 cardiac abnormality. They had severely enlarged atrium and ventricles with distorted shape and not clearly defined border (Fig. 4c, d).

\section{Evaluation of cardiovascular endpoints}

In the embryos with structural cardiac defect, we also looked for several other cardiovascular endpoints at 48 hpf (Table 3). We observed that most of these embryos also had pericardial edema and decreased or absent blood circulation. Haemorrhage occurred only in few embryos injected with caffeine and theophylline, while thrombosis was absent in all the drug injected embryos.

To see the long term fate of the embryos with cardiovascular alterations, one concentration of each drug was injected in the BMP transgenic zebrafish embryos and tracked till $120 \mathrm{hpf}$. The embryos with structural cardiac defects either died or deteriorated further by $120 \mathrm{hpf}$.
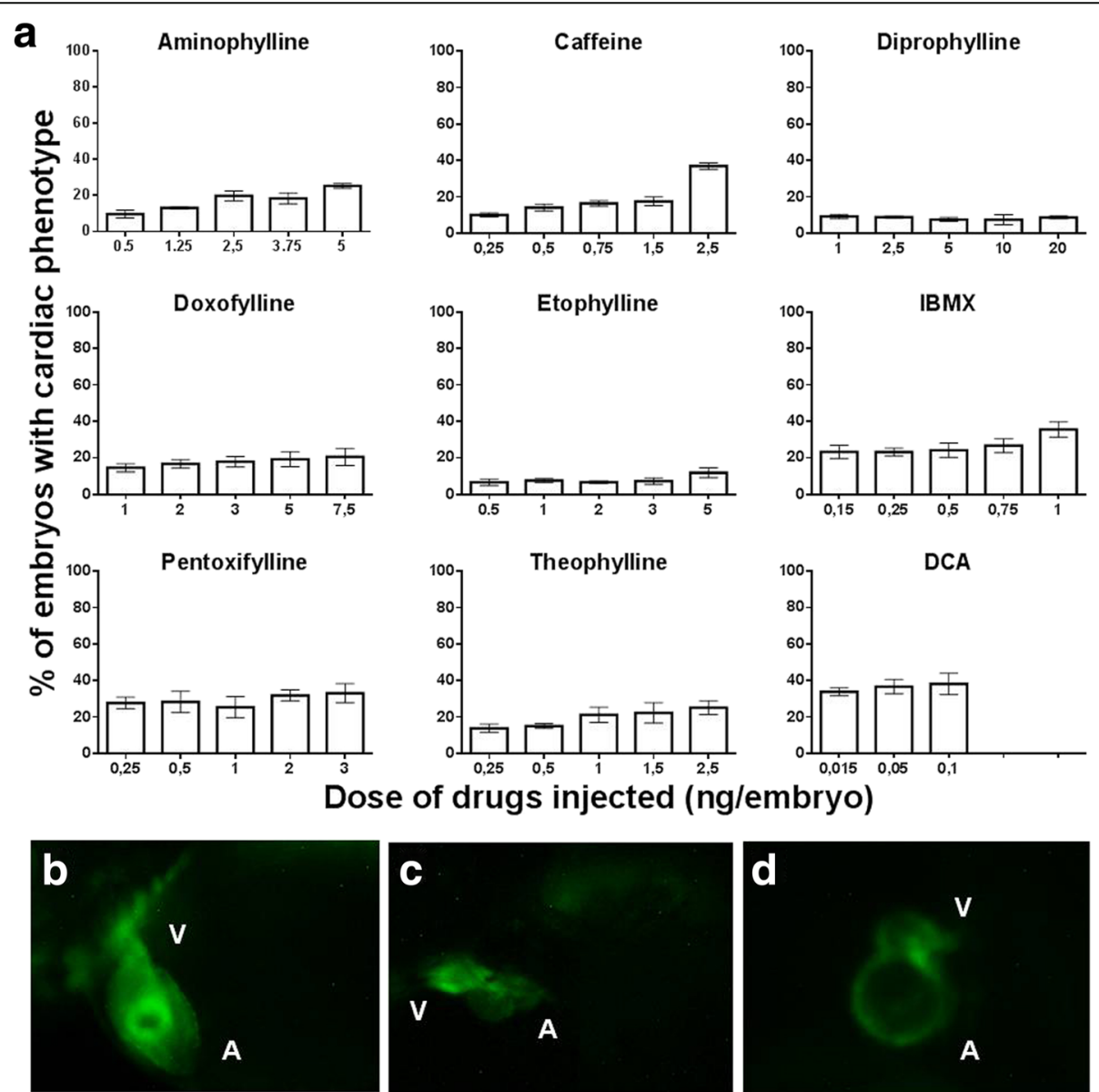

Fig. 4 Structural alteration of the heart at 48 hpf. a Percentage of embryos with structural cardiac abnormality at 48 hpf after microinjection with eight methylxanthines at 1-2 cell stage. Embryos injected with DCA were the positive controls. At least 100 embryos were injected for each drug and the results are the mean \pm SD of three independent experiments. $\mathbf{b}$ Representative picture of normal heart morphology from lateral view. $\mathbf{c}$ and (d) Representative pictures of grade 2 cardiac abnormality observed in methylxanthine treated zebrafish embryos at 48 hpf. Both atrium and ventricle are compressed and not well defined between chambers $(\mathbf{b})$ or atrium and ventricle are severely enlarged, misshaped and not well defined (c). A- Atrium; V-Ventricle 
Table 3

\begin{tabular}{|c|c|c|c|c|c|c|}
\hline \multirow{2}{*}{ Methylxanthine } & \multirow[b]{2}{*}{ Dose } & \multicolumn{5}{|c|}{ Cardiovascular endpoints } \\
\hline & & Pericardial edema & Decreased blood flow & Absent blood flow & Thrombosis & Hemorrhage \\
\hline \multirow[t]{2}{*}{ Aminophylline } & LC & $2 \%$ & $1 \%$ & $1 \%$ & 0 & 0 \\
\hline & $\mathrm{HC}$ & $14 \%$ & $16 \%$ & $6 \%$ & 0 & $2 \%$ \\
\hline \multirow[t]{2}{*}{ Caffeine } & LC & $0.01 \%$ & $0.01 \%$ & 0 & 0 & 0 \\
\hline & $\mathrm{HC}$ & $12 \%$ & $6.5 \%$ & $9.8 \%$ & 0 & $5.4 \%$ \\
\hline \multirow[t]{2}{*}{ Diprophylline } & LC & $0.01 \%$ & $0.01 \%$ & $0.01 \%$ & 0 & 0 \\
\hline & $\mathrm{HC}$ & $7.8 \%$ & $4.95 \%$ & $2.94 \%$ & 0 & $0.01 \%$ \\
\hline \multirow[t]{2}{*}{ Doxofylline } & LC & $4 \%$ & $4 \%$ & $12 \%$ & 0 & 0 \\
\hline & $\mathrm{HC}$ & $13.7 \%$ & $8.21 \%$ & $11 \%$ & 0 & 0 \\
\hline \multirow[t]{2}{*}{ Etofylline } & LC & $0.8 \%$ & $3 \%$ & $2.5 \%$ & 0 & 0 \\
\hline & $\mathrm{HC}$ & $10.2 \%$ & $5.6 \%$ & $7.8 \%$ & 0 & 0 \\
\hline \multirow[t]{2}{*}{ IBMX } & LC & $3.8 \%$ & 0 & $0.03 \%$ & 0 & 0 \\
\hline & $\mathrm{HC}$ & $18 \%$ & $12.5 \%$ & $15.3 \%$ & 0 & $0.01 \%$ \\
\hline \multirow[t]{2}{*}{ Pentoxifylline } & LC & 0 & 0 & 0 & 0 & 0 \\
\hline & $\mathrm{HC}$ & $8.5 \%$ & $8.5 \%$ & $5.7 \%$ & 0 & 0 \\
\hline \multirow[t]{2}{*}{ Theophylline } & LC & $0.01 \%$ & $0.01 \%$ & 0 & 0 & 0 \\
\hline & $\mathrm{HC}$ & $17 \%$ & $9.2 \%$ & $13.8 \%$ & 0 & $7.7 \%$ \\
\hline
\end{tabular}

Affected endpoints of cardiovascular system at $48 \mathrm{hpf}$. The table illustrate the percentage of embryos with a particular endpoint affected at the lowest and highest tested concentration

$L C$ lowest concentration, $H C$ highest concentration

\section{Functional alteration}

Heart rate in the normal looking embryos was measured at $48 \mathrm{hpf}$ to assess the functional alteration of cardiac system. As methylxanthines are adenosine receptor antagonist, we expected to observe an increase in heart rate in the treated embryos if they were affected at functional level. Ten embryos were randomly selected for each concentration of each drug and the heart beat was counted. Embryos treated in sterile water were used as a negative control and metoprolol treated embryos were used as an internal control.

Our results showed that all the methylxanthines with the exception of doxofylline caused a significant increase in heart rate as compared to controls (Fig. 5). Doxofylline did not alter the heart rate whereas metoprolol significantly decreased the heart rate.

To see if the functional effect of these drugs in the embryos was a long lasting effect, the heart rate was tracked every $24 \mathrm{~h}$ from $48 \mathrm{hpf}$ till $120 \mathrm{hpf}$ in the BMP transgenic zebrafish embryos. We found that there was no significant difference in the heart rate in the treated and control embryos at 72, 96 and $120 \mathrm{hpf}$ (Additional file 1).

\section{Discussion}

\section{Embryotoxicity and general Teratogenicity}

In the present study, we showed that if the exposure happens during the early phase of cellular differentiation, a single dose of methylxanthines might be sufficient to induce embryotoxicity and teratogenicity. Almost all the tested methylxanthines induced embryotoxic and teratogenic effects of varying intensity. Based on their potency, these drugs could be classified into three groups: highly toxic and teratogenic, toxic and teratogenic at higher doses and non toxic and teratogenic. Caffeine, IBMX, pentoxifylline and theophylline were highly toxic and teratogenic whereas aminophylline doxofylline and etophylline induced toxicity and teratogenicity but required higher doses. On the other hand, diprophylline induced minimal teratogenicity and toxicity which was comparable to that observed in control-injected embryos. The potency of these drugs in zebrafish embryos was also similar to their potency in human and other animal models. Indeed, studies on humans and other vertebrates have shown that etophylline, doxofylline and diprophylline have lesser side effects than other methylxanthines [12, 35]. Also, in vivo and in vitro studies have shown theophylline to be more potent than etophylline ( 7 fold more potent), diprophylline (100 fold more potent) [35] and doxofylline (10 fold more potent) [36]. Moreover drugs like doxophylline and diprophylline have fewer side effects as compared to other methylxanthines such as theophylline [10, 12]. Overall, our results showed that a single dose of these drugs could be embryotoxic and teratogenic in the zebrafish embryos with potencies comparable to the other higher animal models. 

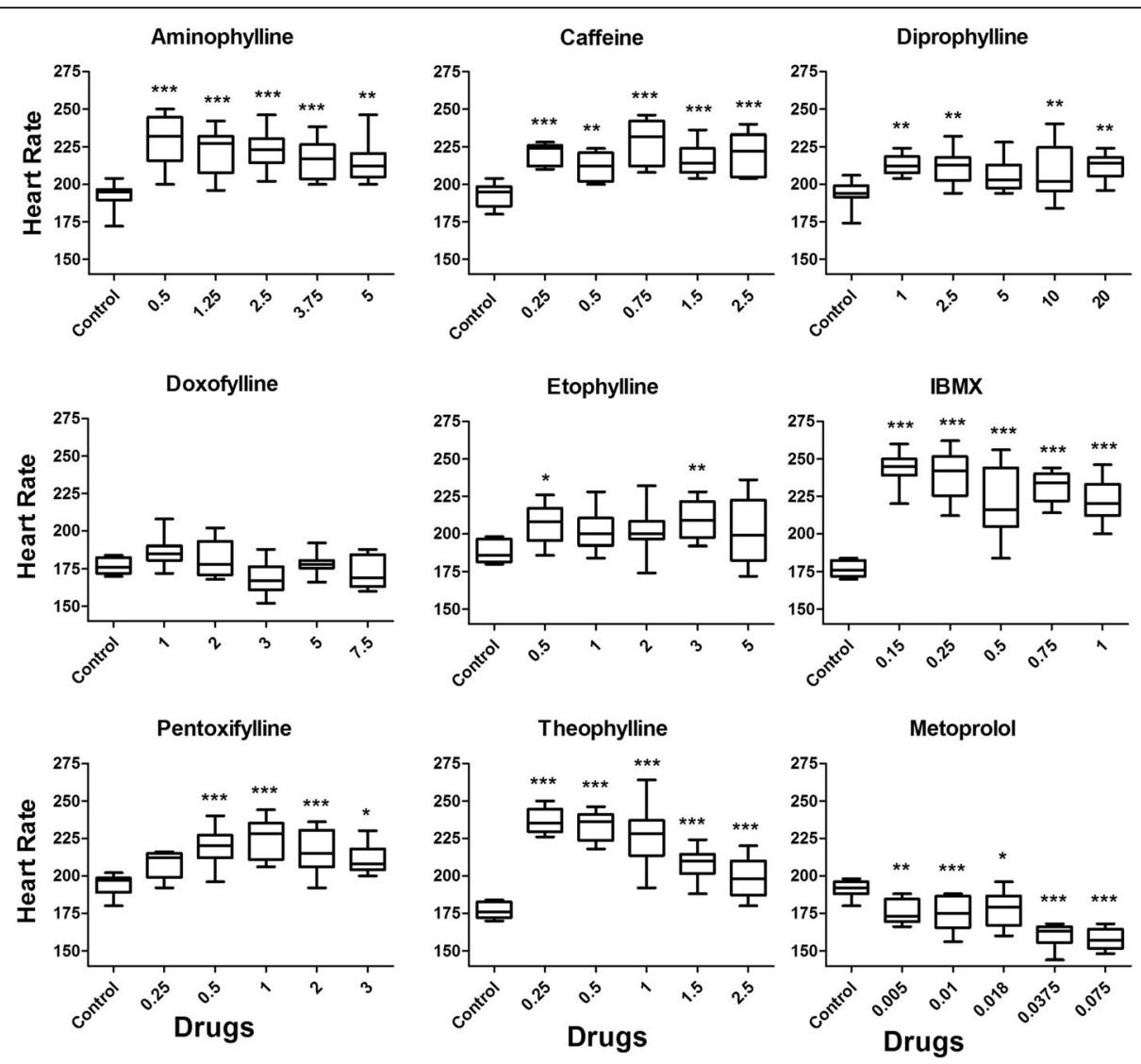

Fig. 5 Heart rate of zebrafish embryos at 48 hpf. Ten normal embryos were randomly selected for each concentration of each drug for counting the heart beat. One way ANOVA with Dunnett's test was used to test the significance of the data. Asterisks indicate that the $p$-value differ significantly from control. ${ }^{* *}=p<0.0005 ;{ }^{* *}=p<0.005 ;{ }^{*}=p<0.05$

\section{Cardiac toxicity and Teratogenicity}

The cardiac toxicity of the methylxanthines can be divided into structural and functional aspects. In our study, structural cardiac defect was not as profound as the functional effect. While the functional effect was present in all the drug treated embryos, structural cardiac defect was present in $<40 \%$ of embryos at the highest concentration with all the methylxanthines. In particular, diprophylline and etophylline induced cardiac defect in less than $10 \%$ of embryos even at the highest injected concentration. Therefore, our results showed two facets of methylxanthine toxicity: structural and functional. While the structural alteration led to death, the functional alteration was transient and mild with the embryos ultimately recovering. One of the major adverse effects of methylxanthines is cardiac toxicity. In human, acute toxicity of methylxanthines cause functional alteration of cardiac physiology such as tachycardia and arrhythmia [1,21] rather than the structural alteration of heart. However, animal studies have shown association of methylxanthines with structural cardiac defect [37, 38] and systematic review has shown cardiovascular malformations as a teratogenic effect of maternal caffeine ingestion in human [39]. Our study showed that methylxanthine induced structural alteration has lower incidence than the functional alteration. However, it could potentially be fatal. In this study, embryos were exposed to doses that fall within the therapeutic range in human. Although, interspecies differences need to be considered before drawing any conclusion, our study indicated that a single exposure of methylxanthines at therapeutic range could induce both functional and structural cardiac disfunction if exposed during early pregnancy before cellular differentiation.

\section{Comparison of cardiac effects with higher vertebrate studies}

Human and animal studies have shown that most of the methylxanthines increases the heart rate $[10,13,15,35$, $40,41]$, whereas doxofylline does not have any effect on it $[10,36,42,43]$. These findings were similar to the results we obtained in the zebrafish embryos where all the methylxanthines increased the heart rate and doxofylline did not have any effect. Therefore, at functional level, there was similarity in the effects of methylxanthines in zebrafish embryos and other animal 
models and human. The ability to respond to both the cardiac stimulant such as methylxanthines and depressant drug such as metoprolol also confirmed the functional maturity of the cardiac system of zebrafish embryos at $48 \mathrm{hpf}$, which is well supported by other zebrafish studies (mentioned in Review [20,44]).

\section{Conclusion}

We investigated the general and cardiac toxicity and teratogenicity of various methylxanthines: aminophylline caffeine, diprophylline, doxofylline, etophylline, IBMX, pentoxifylline and theophylline.

Our results showed that barring diprophylline, a single exposure of these drugs before cellular differentiation could lead to embryotoxicity and general teratogenicity together with the structural and functional alteration of the cardiovascular system. Of all the tested drugs, diprophylline appeared to be safer with fewer incidences of embryotoxicity, general and cardiac teratogenicity $(<10 \%$ of all injected embryos).

\section{Additional file}

Additional file 1: Heart rate of BMP transgenic zebrafish embryos [ $\operatorname{tg}(B m p: E G F P)]$ at 48, 72, 96 and $120 \mathrm{hpf}$ after microinjection with one dose of each drug. The doses selected were: aminophylline $2.5 \mathrm{ng}$, caffeine: $0.75 \mathrm{ng}$, diprophylline $5 \mathrm{ng}$, etophylline $3 \mathrm{ng}$, theophylline $2 \mathrm{ng}$, IBMX $0.5 \mathrm{ng}$, pentoxifylline $1 \mathrm{ng}$, theophylline $1 \mathrm{ng}$. For each drug, ten normal embryos were randomly selected for counting the heart beat. One way ANOVA with Dunnett's test was used to test the significance. Asterisk indicates that the $p$-value differ significantly from control. (JPEG $1268 \mathrm{~kb}$ )

\section{Abbreviations}

DCA: 3, 4-dichloroaniline; hpf: hours post fertilization; IBMX: 3-isobutyl-1methylxanthine; LD: Lethal dose; ng: nanogram

\section{Acknowledgements}

Not applicable

\section{Funding}

This work was supported by Research Institutional Grant from the University of Brescia and by Agenzia Italiana del Farmaco AIFA (Project PHARM-Q).

\section{Availability of data and materials}

All data generated or analysed during this study are included in this published article and its Additional file 1.

\section{Authors' contributions}

$R M B, D Z$ and $M M$ designed the study. RMB and DZ performed the experiments. RMB, DZ, MG, DF and MM analyzed and interpreted the data. RMB drafted the manuscript with help of DZ, MG and DF. MM revised the manuscript. All authors read and approved the final manuscript.

\section{Ethics approval and consent to participate}

Zebrafish were maintained and used in accordance with the Italian and European rules on animal use following protocols approved by the local Committee (OPBA protocol nr 211B5.24) and authorized by the Ministry of Health (authorization number 393/2017-PR).

\section{Consent for publication}

Not applicable

\section{Competing interests}

Maurizio Memo is an editorial board member (Associate Editor) of BMC Pharmacology and Toxicology. The other authors declare no conflict of interest.

\section{Publisher's Note}

Springer Nature remains neutral with regard to jurisdictional claims in published maps and institutional affiliations.

\section{Author details}

${ }^{1}$ Department of Molecular and Translational Medicine, University of Brescia, Viale Europa, 11, 25123 Brescia, Italy. ${ }^{2}$ Clinical Chemistry Laboratory, ASST Spedali Civili di Brescia, 25123 Brescia, Italy.

Received: 31 July 2017 Accepted: 3 November 2017

Published online: 15 November 2017

\section{References}

1. Monteiro J, Alves M, Oliveira P, Silva B. Structure-bioactivity relationships of Methylxanthines: trying to make sense of all the promises and the drawbacks. Molecules. 2016:21:974.

2. Musgrave IF, Farrington RL, Hoban C, Byard RW. Caffeine toxicity in forensic practice: possible effects and under-appreciated sources. Forensic Sci Med Pathol. 2016;12:299-303.

3. Szentmiklósi AJ, Cseppentō A, Gesztelyi R, Zsuga J, Körtvély A, Harmati G, Nánási PP. Xanthine derivatives in the heart.Blessed or cursed? Curr Med Chem. 2011;18:12.

4. Talik P, Krzek J, Ekiert RJ. Analytical techniques used for determination of Methylxanthines and their analogues-recent advances. Sep Purif Rev. 2012:41:1-61.

5. Franco R, Oñatibia-Astibia A, Martínez-Pinilla E. Health benefits of Methylxanthines in cacao and chocolate. Nutrients. 2013;5:4159-73.

6. Sotelo A, Soleri D, Wacher C, Sánchez-Chinchillas A, Argote RM. Chemical and nutritional composition of Tejate, a traditional maize and cacao beverage from the central valleys of Oaxaca, Mexico. Plant Foods Hum Nutr. 2012:67:148-55.

7. Barnes PJ. Theophylline. Pharmaceuticals (Basel). 2010;3:725-47.

8. McCarty MF, O'Keefe JH, DiNicolantonio JJ. Pentoxifylline for vascular health: a brief review of the literature. Open Heart. 2016;3:e000365.

9. Millar D, Schmidt B. Controversies surrounding xanthine therapy. Semin Neonatol. 2004;9:239-44

10. Page CP. Doxofylline: a "Novofylline". Pulm Pharmacol Ther. 2010;23:231-4.

11. Riffo-Vasquez Y, Man F, Page CP. Doxofylline, a novofylline inhibits lung inflammation induced by lipopolysacharide in the mouse. Pulm Pharmacol Ther. 2014:27:170-8.

12. Stablein JJ, Samaan SS, Bukantz SC, Lockey RF. Pharmacokinetics and bioavailability of three Dyphylline preparations. Eur J Clin Pharmacol. 1983;25:281-3.

13. Ujházy E, Onderová E, Horáková M, Bencova E, Durišová M, Nosál' R, Balonová T, Zeljenková D. Teratological study of the hypolipidaemic drugs etofylline clofibrate (VULM) and fenofibrate in Swiss mice. Pharmacol Toxicol. 1989;64:286-90

14. Tarka Jr. SM: The toxicology of cocoa and methyxanthines: a review of literature. CRC Crit Rev Toxicol. 1982; 9(4):275-312.

15. Ellis EF. Theophylline toxicity. J Allergy Clin Immunol. 1985;76:297-301.

16. Holmgren P, Nordén-Pettersson L, Ahlner J. Caffeine fatalities—four case reports. Forensic Sci Int. 2004;139:71-3.

17. Kerrigan $\mathrm{S}$, Lindsey T. Fatal caffeine overdose: two case reports. Forensic Sci Int. 2005;153:67-9.

18. Suárez-Peñaranda JM, Rico-Boquete R, López-Rivadulla M, Blanco-Pampín J, Concheiro-Carro L. A fatal case of suicidal pentoxifylline intoxication. Int J Legal Med. 1998;111:151-3.

19. Lam A, Newhouse MT. Management of Asthma and Chronic Airflow Limitation. Chest. 1990;98:44-52.

20. McGrath P, Li CQ. Zebrafish: a predictive model for assessing drug-induced toxicity. Drug Discov Today. 2008;13:394-401.

21. Menke NB, Walsh SJ, King AM. Cardiotoxicodynamics: toxicity of cardiovascular Xenobiotics. Emerg Med Clin North Am. 2015;33:563-95.

22. Avci S, Sarikaya R, Buyukcam F. Death of a young man after overuse of energy drink. Am J Emerg Med. 1624;2013(31):e1623-4. 
23. Forman J, Aizer A, Young CR. Myocardial infarction resulting from caffeine overdose in an anorectic woman. Ann Emerg Med. 1996;29:178-80.

24. Basnet RM, Guarienti M, Memo M. Zebrafish embryo as an in vivo model for behavioral and pharmacological characterization of Methylxanthine drugs. Int J Mol Sci. 2017;18:596.

25. Gianoncelli A, Bonini SA, Bertuzzi M, Guarienti M, Vezzoli S, Kumar R, Delbarba A, Mastinu A, Sigala S, Spano P, et al. An integrated approach for a structural and functional evaluation of Biosimilars: implications for erythropoietin. BioDrugs. 2015;29:285-300.

26. Guarienti M, Gianoncelli A, Bontempi E, Moscoso Cardozo S, Borgese L, Zizioli D, Mitola S, Depero LE, Presta M. Biosafe inertization of municipal solid waste incinerator residues by COSMOS technology. J Hazard Mater. 2014:279:311-21.

27. Mueller F, Ali S, HGJv M, Richardson MK. Large-Scale Assessment of the Zebrafish Embryo as a Possible Predictive Model in Toxicity Testing. PLoS One. 2011;6:e21076.

28. Ducharme NA, Reif DM, Gustafsson J-A, Bondesson M. Comparison of toxicity values across zebrafish early life stages and mammalian studies: implications for chemical testing. Reprod Toxicol. 2015:55:3-10.

29. Lawrence $C$. The husbandry of zebrafish (Danio Rerio): a review. Aquaculture. 2007;269:1-20.

30. Isogai S, Lawson ND, Torrealday S, Horiguchi M, Weinstein BM. Angiogenic network formation in the developing vertebrate trunk. Development. 2003;130:5281-90.

31. Langheinrich $U$, Vacun $G$, Wagner $T$. Zebrafish embryos express an orthologue of HERG and are sensitive toward a range of QT-prolonging drugs inducing severe arrhythmia. Toxicol Appl Pharmacol. 2003;193:370-82.

32. Thisse C, Zon LI. Organogenesis-heart and blood formation from the Zebrafish point of view. Development. 2002;295:457-62.

33. Collery RF, Link BA. Dynamic smad-mediated BMP signaling revealed through transgenic zebrafish. Dev Dyn. 2011;240:712-22.

34. Panzica-Kelly JM, Zhang CX, Danberry TL, Flood A, DeLan JW, Brannen KC, Augustine-Rauch KA. Morphological score assignment guidelines for the dechorionated zebrafish teratogenicity assay. Birth Defects Res B Dev Reprod Toxicol. 2010;89:382-95.

35. Zuidema J, Merkus FWHM. Pharmacokinetics and pharmacodynamics of diprophylline. Pharmaceutisch Weekblad, Scientific Ed. 1981;3:221-31.

36. Cirillo R, Grossi E, Franzone JS. Doxofylline, an adenosine-nonblocking xanthine, does not induce cardiostimulant effects. Res Commun Chem Pathol Pharmacol. 1989;65:21-34.

37. Bruyere HJ Jr, Michaud BJ, Gilbert EF, Folts JD. The effects of cardioteratogenic doses of caffeine on cardiac function in the 3-day chick embryo. J Appl Toxicol. 1987;7:197-203.

38. Bruyere HJ Jr, Nishikawa T, Uno H, Gilbert JE, Gilbert EF. Pulmonary stenosis with ventricular septal defect, common aorticopulmonary trunk, and dextroposition of the aorta: morphologic and qualitative physiologic effects in caffeine-treated chick embryos. Teratology. 1986;33:119-26.

39. Feng Y, Yu D, Yang L, Da M, Wang Z, Lin Y, Ni B, Wang S, Mo X. Maternal lifestyle factors in pregnancy and congenital heart defects in offspring review of the current evidence. Ital J Pediatr. 2014:40:85.

40. Nathan JR, Lakshmanan G, Michael FM, Seppan P, Ragunathan M. Expression of adenosine receptors and vegf during angiogenesis and its inhibition by pentoxifylline-a study using zebrafish model. Biomed Pharmacother. 2016;84:1406-18.

41. Yeh CH, Liao YF, Chang CY, Tsai JN, Wang YH, Cheng CC, Wen CC, Chen YH. Caffeine treatment disturbs the angiogenesis of zebrafish embryos. Drug Chem Toxicol. 2012;35:361-5.

42. Goldstein MF, Chervinsky P. Efficacy and safety of doxofylline compared to theophylline in chronic reversible asthma - a double-blind randomized placebo-controlled multicentre clinical trial. Med Sci Monit. 2002;8:CR297-304.

43. Thisse C, Zon LI. Organogenesis - heart and blood formation from the Zebrapsh point of view. Science. 2002;295:457-62.

44. Lawson ND, Weinstein BM. In vivo imaging of embryonic vascular development using transgenic Zebrafish. Dev Biol. 2002;248:307-18.

\section{Submit your next manuscript to BioMed Central and we will help you at every step:}

- We accept pre-submission inquiries

- Our selector tool helps you to find the most relevant journal

- We provide round the clock customer support

- Convenient online submission

- Thorough peer review

- Inclusion in PubMed and all major indexing services

- Maximum visibility for your research

Submit your manuscript at www.biomedcentral.com/submit
Biomed Central 